\title{
Fast Non-Blocking Atomic Commit: An Inherent Trade-off
}

\author{
Partha Dutta, Rachid Guerraoui, Bastian Pochon* \\ Distributed Programming Laboratory, EPFL \\ CH-1015 Lausanne, Switzerland
}

\begin{abstract}
This paper investigates the time-complexity of the non-blocking atomic commit (NBAC) problem in a synchronous distributed model where $t$ out of $n$ processes may fail by crashing. We exhibit for $t \geq 3$ an inherent trade-off between the fast abort property of NBAC, i.e., aborting a transaction as soon as possible if some process votes "no," and the fast commit property, i.e., committing a transaction as soon as possible when all processes vote "yes" and no process crashes. We also give two algorithms: the first satisfies fast commit and a weak variant of fast abort, whereas the second satisfies fast abort and a weak variant of fast commit.
\end{abstract}

Key words: Distributed algorithms, complexity, atomic commit

\section{Introduction}

The synchronous model. We consider a set $\Pi=\left\{p_{1}, p_{2}, \ldots, p_{n}\right\}(n \geq 3)$ of processes in a synchronous crash-stop model [5]. ${ }^{1}$ The processes may fail by crashing and do not recover from a crash. Any process that does not crash in a run (any execution of an algorithm) is said to be correct in that run; otherwise the process is said to be faulty. In any given run, at most $t<n$ processes may crash, and we denote by $f$ the effective number of processes that crash in that run. The processes proceed in rounds. Each round consists of two phases: (a) in the send phase, all processes (that did not crash) send messages to all processes; (b) in the receive phase, the processes receive the messages sent in the send phase of that round and update their local states. If some process $p_{i}$ completes the send phase of the round, every process that completes the receive phase of the round receives the message sent by $p_{i}$ in

\footnotetext{
* Corresponding author: Bastian.Pochon@epfl.ch

1 We refer the reader to [5] for details on the model.
} 
the send phase. If $p_{i}$ crashes during the send phase, then any subset of the processes might not receive the message sent by $p_{i}$ in that round.

The Non-blocking Atomic Commit Problem. In the non-blocking atomic commit problem $[1,6]$ (NBAC), each process is supposed to cast a vote, either 0 or 1, proposing to either abort or commit a distributed transaction. Each process is supposed to eventually decide ${ }^{2}$ on either 0 (abort the transaction) or 1 (commit the transaction), such that the following properties are satisfied: (uniform agreement) no two processes decide differently, (termination) every correct process eventually decides, (abort validity) 0 is the only possible decision if some process proposes 0 , and (commit validity) 1 is the only possible decision if every process is correct and proposes 1 .

The abort validity property of NBAC states that, if any process proposes 0 , then 0 is the only possible decision value. This leads to an interesting observation: if a process $p_{i}$ receives a message from any process that proposes 0 , then $p_{i}$ can immediately decide 0 . Clearly, there is an algorithm which ensures a global decision ${ }^{3}$ by round 1 in any run in which some process proposes 0 (no matter how many crashes occur in that run). This property, which we call fast abort, allows the processes to quickly retry committing a transaction in case of a "logical" abort. ${ }^{4}$

On the other hand we would also like to commit a transaction as fast as possible when all processes propose 1 . In $[2,4]$, it is shown that in runs with at most $f$ crashes $(0 \leq f \leq t), \min (f+2, t+1)^{5}$ is a lower bound for a global decision. An algorithm that achieves this bound, for $0 \leq f \leq t$, is said to be early deciding. We say that a NBAC algorithm satisfies the fast commit property, if it globally decides by round 2 in every run in which all processes propose 1 and no process crashes. Note that early decision implies fast commit.

Contribution. Interestingly, for $t \geq 3$, we show that fast abort is incompatible with fast commit. More precisely, while fast abort and fast commit can both be individually achieved (as we discuss later in the paper), we prove that no single NBAC algorithm can have both properties. We also present two NBAC algorithms, each of these satisfying one of the properties and a weaker form of the other one. We say in this context that a NBAC algorithm satisfies weak fast abort if it globally decides by round 2 in every run in which some process proposes 0 , and a NBAC algorithm satisfies weak fast commit if it globally decides by round 3 in every run in which all processes propose

$\overline{2}$ Throughout this paper, our bounds are for decision events, not halting events.

3 A run globally decides in round $k$ if every process that decides in that run, decides by round $k$, and some process decides in round $k$.

4 I.e., some process proposes 0 . This could occur for instance because of a concurrency control problem.

5 For the sake of brevity we are being slightly imprecise here; the lower bound really is $f+2$ for $f \leq t-2$, and $f+1$ for $f \geq t-1$. The special case is $f=t-1$. 
1 , and no process crashes. Our first algorithm satisfies fast commit and weak fast abort, and our second algorithm satisfies fast abort and weak fast commit. Additionally, both algorithms match the bounds of $[2,4]$ for the runs with process crashes, namely, they both globally decide in $\min (f+2, t+1)$ rounds in runs with at most $f$ crashes, provided $f \geq 1$.

\section{Incompatibility of Fast Commit and Fast Abort}

As previously mentioned, it is possible to globally decides by round 1 in every run in which some process proposes 0 . However, observe that if a process $p_{i}$ is required to decide in round 1 in any run in which some process proposes 0 , then $p_{i}$ has to decide 0 in round 1 if $p_{i}$ does not receive the round 1 message from any other process $p_{j}$, because $p_{i}$ does not know whether $p_{j}$ proposed 0 or 1.

Proposition 1 For $3 \leq t \leq n-1$, no NBAC algorithm can satisfy both the fast abort and the fast commit properties.

Proof. Consider by contradiction a NBAC algorithm $A$ which satisfies both fast abort and fast commit. We exploit indistinguishability between five different runs of $A$, and derive a contradiction.

1. In run $R 1$, process $p_{1}$ proposes 0 , and all other processes propose 1 . Process $p_{1}$ crashes before sending any message in round 1 . By abort validity, the only possible decision in this run is 0 . By fast abort, every process distinct from $p_{1}$ decides 0 at the end of round 1 , in particular $p_{2}$.

2. Run $R 2$ starts from the initial configuration in which all processes propose 1 (including $p_{1}$ ). Process $p_{1}$ crashes in round 1 after sending a message to all processes but $p_{2}$. Clearly, $p_{2}$ cannot distinguish $R 1$ from $R 2$. Thus $p_{2}$ decides 0 at the end of round 1 in $R 2$.

3. Run $R 3$ is identical to $R 2$, except that $p_{2}$ now crashes at the beginning of round 2 , before sending any message in round 2 , and $p_{3}$ crashes at the beginning of round 3. All remaining processes are correct. Clearly, at the end of round 1, R2 and $R 3$ are indistinguishable for $p_{2}$, and hence, $p_{2}$ decides 0 at the end of round 1 in $R 3$, and then crashes.

4. Run $R 4$ is failure-free, starting from the initial configuration in which all processes propose 1 . By fast commit, and commit validity, all processes decide 1 at the end of round 2 in $R 4$, in particular $p_{3}$.

5. Finally, run $R 5$ is similar to $R 4$, but processes $p_{1}$ and $p_{2}$ crash in the send phase of round 2 , such that both processes send a message to only $p_{3}$ in round 
2 , and process $p_{3}$ crashes at the beginning of round 3. Clearly, $R 4$ and $R 5$ are indistinguishable for $p_{3}$ at the end of round 2. Thus $p_{3}$ decides 1 at the end of round 2 , and then crashes.

In $R 3$, process $p_{2}$ decides 0 and crashes. In $R 5$, process $p_{3}$ decides 1 and crashes. Runs $R 3$ and $R 5$ are however indistinguishable for all processes distinct from $p_{1}, p_{2}$, and $p_{3}$. To see why, observe that $R 3$ and $R 5$ are different only at $p_{1}$ and $p_{2}$ at the end of round 1 , and $p 1$ and $p_{2}$ send messages only to $p_{3}$ in round 2 . None of the three processes send any messages after round 2. This contradicts uniform agreement.

In the next section, we circumvent this incompatibility by weaking one of the properties when $t \geq 3$. We give two algorithms: the first algorithm satisfies weak fast abort and fast commit, whereas the second algorithm satisfies fast abort and weak fast commit. For $t \leq 2$, it is possible to design an NBAC algorithm that satisfies both fast commit and fast abort: we give that algorithm in $[3]$.

\section{Fast NBAC Algorithms}

In this section we assume that $t \geq 3$. We first give a NBAC algorithm in Fig. 1, which satisfies fast commit and weak fast abort. The algorithm is called FCWFA. It is a flooding algorithm, optimized for the fast commit and the weak fast abort properties, and the special case where $f=t-1$. In round 1 , the processes exchange their estimate est, initialized to their proposal value, and try to adjust their estimate in anticipation of a weak fast abort: if a process does not receive est $=1$ from all processes, it changes its estimate to 0 , as it might be the case that some process proposed 0 . In round 2, after exchanging their estimate, the processes decide 0 if they are certain that any other process will either decide 0 or continue with a 0 estimate. Otherwise, the processes decide at the end of round 2 if they notice a failure-free run. From round 2 on, each process $p_{i}$ records, in a set Halt $t_{i}$, the identity of the processes known to have crashed. In the next rounds, processes exchange their estimate with each other, and update their set Halt in $_{i}$ with the identity of the processes from which no message has been received. A process $p_{i}$ decides in a round $r \geq 2$ whenever its set Halt $_{i}$ does not contain more than $r-2$ processes.

Interestingly, FCWFA can easily be adapted to a binary uniform consensus algorithm reaching all known global decision lower bounds, by removing lines $7,8,19$, and 20. Additionally, for the case $f=t-1$, the resulting uniform consensus algorithm, can be viewed as a drastic simplification of the Tree $_{t}$ algorithm of [2].

With FCWFA, every process which decides, decides by round $f+2$, for $f \leq$ 


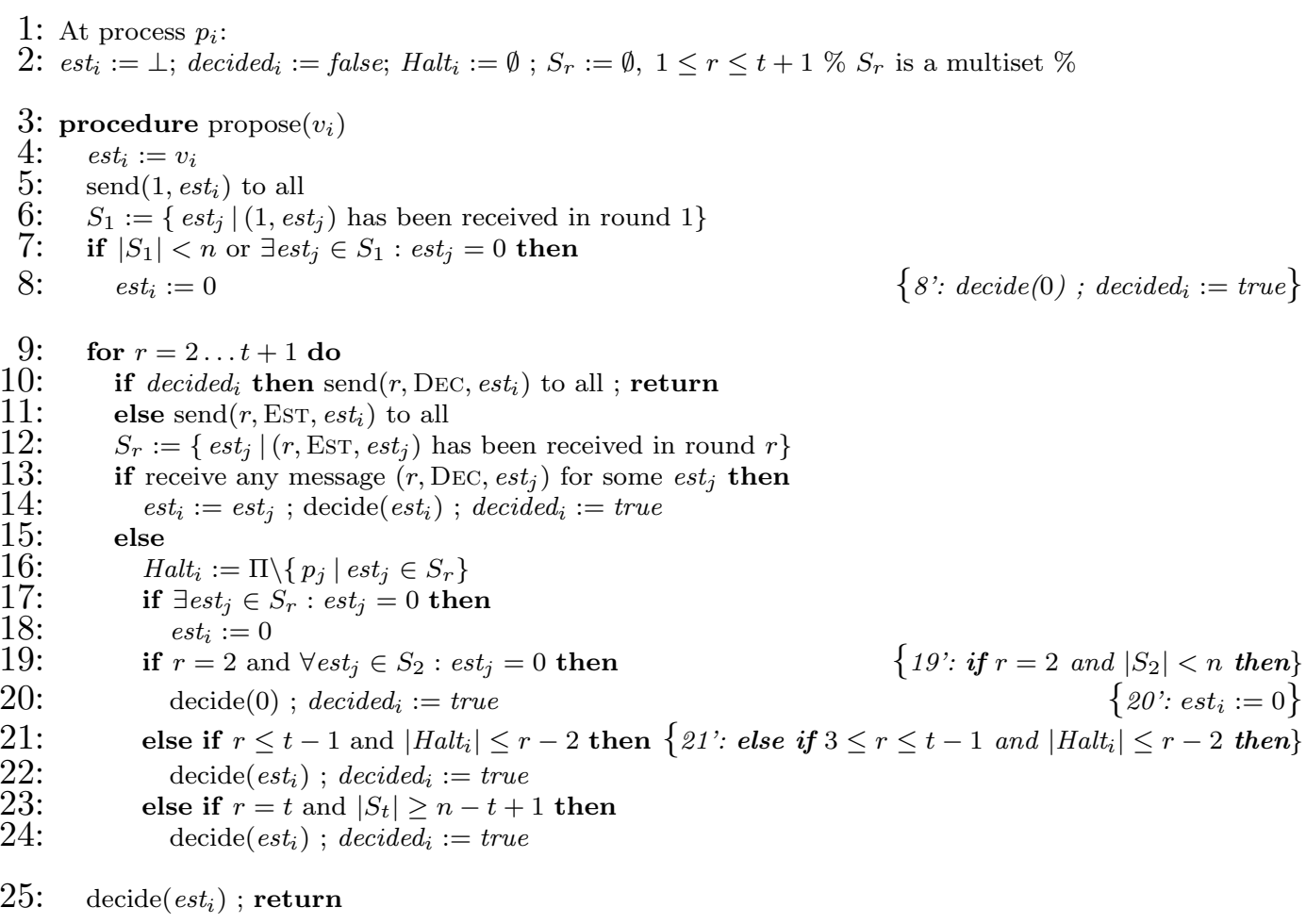

Fig. 1. A fast commit, weakly fast abort, early deciding NBAC algorithm (FCWFA). Replacing line 8,19,20 and 21 with $8^{\prime}, 19^{\prime}, 20^{\prime}$ and $21^{\prime}$ gives a fast abort, weakly fast commit NBAC algorithm (FAWFC).

$t-2$, or round $f+1$, for $f \geq t-1$, in every run where there are at most $f$ processes that crash (early deciding). For an intuition of why FCWFA is faster when $f=t-1$ (vs. $f \leq t-2$ ), consider a run in which no process has decided by round $t-1$. At the end of round $t-1$, two processes have different estimates only if there remains at most a single process that may crash (that is, $f \geq t-1)$. Hence, any process can decide on its estimate at the end of round $t$, if it receives $n-t+1$ messages in round $t$. For the sake of clarity, we omit the obvious optimization where any process which proposes 0 can decide 0 before taking any step in the algorithm.

Interestingly, a slight modification of FCWFA results in a second NBAC algorithm that satisfies weak fast commit and fast abort properties. This second algorithm is called FAWFC. The corresponding modifications are shown between brackets directly in Fig. 1.

We prove the correctness of the algorithms and their complexity properties. In both algorithms, variable $S_{r}$, for $1 \leq r \leq t+1$, denotes sets which can hold duplicate values at the same time. In the following proofs, we denote the local copy of a variable var at process $p_{i}$ by $v_{a r}$, and the value of $v_{a r}$ at the end of round $r$ by $\operatorname{var}_{i}^{r}$. We call a message carrying an estimate $e s t=1$ a commit message, and similarly, a message carrying an estimate est $=0$ an 
abort message. We denote by crashed ${ }^{r}$ the set of processes that crash before completing round $r$. We first prove two general claims which hold for both algorithms.

Claim 2 In FCWFA and FAWFC, if no process has decided by round $r-1 \geq$ 1 and at the end of round $r$ two distinct processes $p_{i}$ and $p_{j}$ are such that est $_{i}^{r} \neq$ est $_{j}^{r}$, then $\mid$ crashed $^{r} \mid \geq r$.

Proof. We prove the claim by induction on the round number. We note that if no process decides by round $r-1$, then processes do not receive any DEC message in round $r$, and hence update their estimate in round $r$. For the base case $r=2$, assume that the conditions of the claim hold, and that, w.l.o.g., $e s t_{i}^{2}=1$ and $e s t_{j}^{2}=0$. It follows that $e s t_{j}^{1}=1$; otherwise, upon receiving the abort message from $p_{j}$ in round $2, p_{i}$ would have changed its est to 0 . In round 2 , since $p_{j}$ changed its est from 1 to $0, p_{j}$ received at least one abort message that $p_{i}$ has not received. Hence some process $p_{k}$ sent an abort message in round 2 and crashed in the send phase of round 2 before sending the message to $p_{i}$. Thus, est $t_{k}^{1}=0$. Furthermore, since est $t_{i}^{2}=1$, est $t_{i}^{1}$ is also 1 , and it follows that $p_{i}$ received commit message from all $n$ processes in round 1 . Since est $t_{k}^{1}=0$ and all process have sent commit messages in round $1, p_{k}$ has received less than $n$ message in round 1 . Thus, some process distinct from $p_{k}$ has crashed in round 1. Hence $\mid$ crashed $^{2} \mid \geq 2$. Assume now the claim for round $r-1$ (induction hypothesis). We prove the claim for round $r$. Suppose that no process decides by round $r$ and consider two distinct processes $p_{k}$ and $p_{l}$ such that $e s t_{k}^{r}=1$ and est $_{l}^{r}=0$. Clearly, est $t_{k}^{r-1}=1$. As both processes completed round $r, p_{k}$ received round $r$ message from $p_{l}$, hence est $r_{l}^{r-1}=1$. Thus there is a process $p_{x}$ which sent an abort message to $p_{l}$ in round $r$, and crashed before sending a round $r$ message to $p_{k}$. Thus, est $t_{x}^{r-1}=0$. Since $e s t_{k}^{k-1}=1$ and $e s t_{x}^{r-1}=0$ and no process has decided by round $r-2$, from induction hypothesis it follows that $\mid$ crashed $^{r-1} \mid \geq r-1$. As $p_{x}$ crashes in round $r, \mid$ crashed $^{r} \mid \geq r$.

Claim 3 In FCWFA and FAWFC, for any round $r \geq 2$ and any process $p_{i}$ that completes round $r$ without receiving a DEC message, crashed $^{r-1} \subseteq$ Halt $_{i}^{r}$.

Proof. Since $p_{i}$ completes round $r$ without receiving a DEC message, it updates Halt $_{i}$ in line 16 . If a process $p_{j}$ crashes by round $r-1$, then $p_{i}$ does not receive round $r$ message from $p_{j}$, and hence, includes $p_{j}$ in Halt $t_{i}$.

The next two propositions assert the correctness and efficiency of FCWFA. (The corresponding proofs for FAWFC can be obtained by straightforward modifications; for space limitation, we give those proofs in [3].)

Proposition 4 FCWFA solves $N B A C$.

Proof. We prove here the termination, commit validity, abort validity, and agreement properties of NBAC in FCWFA. 
Termination. All correct processes decide by round $t+1$, and no process blocks in any round.

Abort-Validity. If any process proposes 0 then, every process that completes round 1, either receives less than $n$ messages or receives at least one abort message, and hence, executes line 8 . Thus, in round 2 , only abort messages are exchanged amongst processes. Every process that completes round 2 executes line 20 and decides 0 .

Commit-Validity. Consider a run in which every process proposes 1 and no process fails. At the end of round 1, every process receives commit messages from $n$ processes, and hence, does not executes line 8 . Thus, in round 2 , only commit messages are exchanged amongst processes. Consequently, processes receive $n$ commit messages in round 2 as well, and for all processes, Halt $^{2}=\emptyset$. Thus every process decides 1 at line 22 .

Uniform Agreement. We consider the lowest round $r$ in which at least one process decides. Let $p_{i}$ be one of the processes that decides in round $r$, say on value $v$. We show that every process that decides in round $r$, decides $v$, and processes that complete round $r$ without deciding, have est $t^{r}=v$. This immediately implies uniform agreement. We consider four cases: (1) $r=2$, (2) $3 \leq r \leq t-1$, (3) $r=t$, and (4) $r=t+1$. (Notice that no process decides in round 1.)

Case 1. Consider the subcase (1a) where $v=1$. Since $p_{i}$ decides 1 , it did not receive any abort message. Furthermore, as $p_{i}$ decides in round $2, \mid$ Halt $_{i}^{2} \mid \leq 0$, i.e., $p_{i}$ received round 2 messages from all processes. In other words, $p_{i}$ received $n$ commit messages in round 2 . Hence, all processes received $n$ commit messages in round 1, and no process crashes before completing round 1. Therefore, only commit messages are sent in round 2 . Thus, no process decides 0 in round 2 , and every process that completes round 2 , has $e s t^{2}=1$. Consider now the subcase (1b) where $v=0$. Thus $p_{i}$ receives only abort messages in round 2 , including from itself. Since $p_{i}$ completes round 2 , any process that completes round 2 , receives the abort message from $p_{i}$. Thus no process can decide 1 in round 2 , and every process that completes round 2 without deciding, changes its est to 0 on receiving the abort message from $p_{i}$.

Case 2. We note that $p_{i}$ must have decided at line 22. (Process $p_{i}$ cannot decide at line 14 because $r$ is the lowest round in which some process decides.) Suppose by contradiction that some process $p_{j}$ decides $1-v$ in round $r$, or completes round $r$ with $e s t^{r}=1-v$. Since both $p_{i}$ and $p_{j}$ complete round $r$, they receive each other's round $r$ messages. If any of them has est $=0$ at the end of round $r-1$, then both processes would have est $t^{r}=0$. Hence, $e s t_{i}^{r-1}=e s t_{j}^{r-1}=1$. Thus in round $r$, some process $p_{x}$ sent an abort message to one of the processes $\left(p_{i}\right.$ or $\left.p_{j}\right)$ and not to the other one. Thus est $t_{x}^{r-1}=0$, and, 
by Claim 2, $\mid$ crashed $^{r-1} \mid \geq r-1$. Thus, at the end of round $r$, by Claim $3,{ }^{6}$ $\mid$ Halt $_{i}^{r} \mid \geq r-1$. A contradiction with the fact that $p_{i}$ decides in line line 22 of round $r$.

Case 3. No process has decided by round $t-1$. If all processes that complete round $t-1$ have the same est, then uniform agreement trivially follows. Suppose two processes have different est at the end of round $t-1$. Then by Claim 2, $\mid$ crashed $^{t-1} \mid \geq t-1$; i.e., there are at most $n-t+1$ processes that complete round $t-1$. Since $p_{i}$ decides in round $r=t$, so $p_{i}$ decides in line 24 and has received at least $n-t+1$ message in round $t$. Thus exactly $n-t+1$ processes complete round $t-1$. If any other process decides in round $t$, it receives the same $n-t+1$ messages as $p_{i}$, and hence, decides $v$. If a process $p_{j}$ completes round $t$ without deciding, then it has received $n-t$ messages in round $t$, and hence, $t$ processes crash by round $t$. Then, $p_{i}$ is a correct processes (as it has completed round $t$ ), and $p_{j}$ receives the DEC message sent by $p_{i}$ in round $t+1$, and decides $v$.

Case 4. If no process decides by round $t$ and two processes have distinct est at the end of round $t+1$, then from Claim $2, \mid$ crashed $^{t+1} \mid \geq t+1$. A contradiction.

Proposition 5 FCWFA satisfies weak fast abort, fast commit, and early decision.

Proof. For weak fast abort, consider a run that starts from an initial configuration where at least one process $p_{i}$ proposes 0 . Every process $p_{j}$ which completes round 1 sets its estimate $e t_{j}$ to 0 at the end of round 1 (because either $p_{j}$ receives $p_{i}$ 's abort message, or $p_{j}$ does not receive any message from $p_{i}$ ). Thus processes receive only abort messages in round 2 . Thus, every process that completes round 2 , decides 0 at that round (line 20).

Notice that, early decision for $f=0$, implies fast commit. We now show that the algorithm satisfies early decision. Suppose, $f \leq t-2$ in a run, and some process $p_{i}$ completes round $f+2$ without deciding. Then $p_{i}$ has not received any DEC message by round $f+2$. We claim that every process in $\mathrm{Halt}_{i}^{f+2}$ is faulty. Suppose otherwise; if some correct process $p_{j}$ is in $\mathrm{Halt}_{i}^{f+2}$, then $p_{j}$ has halted after deciding, and it has sent a DEC message in round $f+2$ or a lower round. Since $p_{i}$ has not received any DEC message by round $f+2$, no correct process is in Halt $_{i}^{f+2}$. Thus $\mid$ Halt $_{i}^{f+2} \mid \leq f$. Thus, in round $f+2, p_{i}$ evaluates the condition in line 21 to true, and decides in line 22. For the case where $f=t-1$, observe that, if $f=t-1$ processes crash in a run, and some process does not decides by round $t=f+1$, then at the end of round $t=f+1$, every process that is not crashed, either receives a DEC message or receives at least

$\overline{6}$ Since $r$ is the lowest round in which some process decides, $p_{i}$ does not receive any DEC message in round $r$. 
$n-t+1$ messages, and hence, decides on its estimate. If $f=t$, clearly, every process that decides, decides by round $f+1=t+1$.

\section{Concluding Remarks}

In the decentralized (non-blocking) three-phase commit (D3PC) algorithm of [6], which is the fastest NBAC algorithm we knew of so far (in terms of number of rounds), all processes decide in round 1 in every failure-free run where some process proposes 0 , and in round 2 in the failure-free run where all processes propose 1 . In D3PC however, no process decides in round 1 in a run where some process proposes 0 and crashes before sending any message. This means, in our terminology, that D3PC satisfies fast commit but not fast abort, which is consistent with our incompatibility result. Moreover D3PC does not satisfy early decision provided $f \geq 1$.

\section{Acknowledgments}

We thank the anonymous reviewers for their suggestions that significantly helped us to improve the presentation of the paper and specifically the algorithms.

\section{References}

[1] Bernstein P.A., Hadzilacos V. and Goodman N., Concurrency Control and Recovery in Database Systems (Addison-Wesley, 1987).

[2] Charron-Bost B. and Schiper A., Uniform Consensus Harder than Consensus, EPFL Technical Report DSC/2000/028 (EPFL, 2000).

[3] Dutta P., Guerraoui R., Pochon B., Fast Non-Blocking Atomic Commit: An Inherent Trade-off, EPFL Technical Report, ID IC/2004/29, School of Computer and Communication Sciences, EPFL, 2003. Available at: http://ic2.epfl.ch/publications/documents/IC_TECH_REPORT_200429.pdf

[4] Keidar I. and Rajsbaum S., On the Cost of Fault-Tolerant Consensus when There are No Faults: a Tutorial, MIT Technical Report MIT-LCS-TR-821 (MIT, 2001). Also Information Processing Letters (IPL), 85(1): 47-52, 2003.

[5] Lynch N., Distributed Algorithms (Morgan Kaufmann, San Francisco, 1996).

[6] Skeen D., Nonblocking commit protocols, ACM SIGMOD International Symposium on Management of Data, pages 133-142, 1981. 